\title{
Obtenção do pó da entrecasca do Juazeiro (Ziziphus joazeiro Mart.) por secagem em
}

\section{camada de espuma}

\author{
Obtaing the powder of the bark from Ziziphus Joazeiro Mart. by foam-mat drying \\ Obtención de polvo de la entrecasca de Ziziphus Joazeiro Mart. por secado em capa de espuma
}

\author{
Mariana Fortini Moreira \\ ORCID: https://orcid.org/0000-0002-9022-4782 \\ Universidade Federal da Paraíba, Brasil \\ E-mail: fortinimariana8@gmail.com \\ Josilene de Assis Cavalcante \\ ORCID: https://orcid.org/0000-0003-1179-1576 \\ Universidade Federal da Paraíba, Brasil \\ E-mail: josy_cavalcante@yahoo.com.br \\ Nagel Alves Costa \\ ORCID: https://orcid.org/0000-0002-1724-2677 \\ Universidade Federal da Paraíba, Brasil \\ E-mail:nagelalves@hotmail.com.br \\ Marcelo Felipe Rodrigues da Silva \\ ORCID: https://orcid.org/0000-0001-7558-9655 \\ Universidade Federal da Paraíba, Brasil \\ E-mail:marcelo.mfrs@itf.ufpb.br
}

\begin{abstract}
Resumo
O juazeiro (Ziziphus joazeiro Mart.), é uma árvore encontrada na região semiárida do Nordeste Brasileiro e é considerado um representante notável da Caatinga. Possui diversas aplicações e um elevado teor de saponina. O objetivo desse trabalho foi estudar a secagem em camada de espuma da entrecasca do Ziziphus joazeiro para obtenção do pó. Foi estudada a influência das variáveis: temperatura de secagem, tempo de agitação e concentração de juazeiro, no tempo final de secagem. Foram avaliadas as características físicas da espuma: estabilidade, densidade, expansão e incorporação de ar. Também foi feita a caracterização físico-química do pó obtido através das análises: atividade de água; solubilidade; teor de sólidos solúveis; $\mathrm{pH}$ e espectros de RMN. As espumas apresentaram boa estabilidade; a densidade diminuiu com a concentração de juazeiro e com o tempo de agitação; maior incorporação de ar para o experimento com maior tempo de agitação. Na secagem, a única variável com influência estatisticamente significativa no intervalo de confiança de $95 \%$ no tempo final de secagem foi a temperatura. Os valores de atividade de água do pó, solubilidade em água e $\mathrm{pH}$ foram considerados na faixa aceitável, o teor de sólidos solúveis do pó obtidos foi considerado baixo. Não foi identificado os sinais característicos de saponinas nos espectros de RMN. Portanto, concluise que secagem em camada de espuma mostrou-se um método satisfatório e viável para a obtenção da entrecasca do juazeiro em pó, e o pó obtido apresentou características que o apontam como um bom agente espumante.
\end{abstract}

Palavras-chave: Juazeiro; Saponinas; RMN.

\begin{abstract}
The juazeiro (Ziziphus joazeiro Mart.), is a tree found in the semiarid region of Northeast Brazil and is considered a notable representative of the Caatinga. It has several applications, and a high saponin content. Therefore, the objective of this work was to study the drying in a foam layer (foam-mat drying) of the Ziziphus joazeiro bark for later obtaining the powder. The influence of the following variables was studied: drying temperature, stirring time and juazeiro concentration, on the final drying time of the bark. And the physical characteristics of the formed foam were evaluated: stability, density, expansion and incorporation of air. The physicochemical characterization of the powder obtained was also carried out through the following analyses: water activity; solubility; soluble solids content; $\mathrm{pH}$ and NMR spectra. The foams showed good stability; the density decreased with the concentration of juazeiro and with the time of agitation; greatest incorporation of air for the experiment with the longest stirring time. In drying, the only variable with a statiscally significant influence on the $95 \%$ confidence interval fot the final drying time was temperature. The values of water activity of the powde and $\mathrm{pH}$ were considered in the acceptable range, the soluble solids content of the pow der obtained was considered low. It was not possible to identify the characteristic signals of saponins in the NMR spectra. Therefore, it is concluded that drying in a foam layer proved to be a satisfactory and viable method to obtain powdered of the bark from Ziziphus joazeiro, and the powder obtained showed characteristics that point to it as a good foaming agent.
\end{abstract}

Keywords: Juazeiro; Saponins; NMR. 


\begin{abstract}
Resumen
El juazeiro (Ziziphus joazeiro Mart.), es un árbol que se encuentra en la región semiárida del noreste de Brasil y es considerado un representante notable de la Caatinga. Tiene varias aplicaciones y un alto contenido en saponinas. El objetivo de este trabajo fue estudiar el secado en una capa de espuma de la corteza de Ziziphus joazeiro para obtener el polvo. Se estudió la influencia de las variables: temperatura de secado, tiempo de agitación y concentración de azufaifo en el tiempo de secado final. Se evaluaron las características físicas de la espuma: estabilidad, densidad, expansión e incorporación de aire. La caracterización fisicoquímica del polvo obtenido también se realizó mediante los siguientes análisis: actividad del agua; solubilidad; contenido de sólidos solubles; Espectros de pH y RMN. Las espumas mostraron buena estabilidad; la densidad disminuyó con la concentración de juazeiro y con el tiempo de agitación; mayor incorporación de aire para el experimento con mayor tiempo de agitación. En el secado, la única variable con influencia estadísticamente significativa en el intervalo de confianza del $95 \%$ para el tiempo de secado final fue la temperatura. Los valores de actividad en agua del polvo, solubilidad en agua y $\mathrm{pH}$ se consideraron en el rango aceptable, el contenido de sólidos solubles del polvo obtenido se consideró bajo. No se identificaron las señales características de las saponinas en los espectros de RMN. Por tanto, se concluye que el secado en capa de espuma resultó ser un método satisfactorio y viable para la obtención de corteza de azufaifa en polvo, y el polvo obtenido presentó características que lo apuntan como un buen agente espumante.
\end{abstract}

Palabras clave: Juazeiro; Saponinas; RMN.

\title{
1. Introdução
}

O juazeiro (Ziziphus joazeiro Mart.) é uma planta pertencente à família das Rhamnaceae encontrada principalmente na região semiárida do Nordeste Brasileiro, e é considerado um representante notável da Caatinga (Lorenzi \& Matos, 2008). Conhecido popularmente como joazeiro, juazeiro ou juá é uma árvore de médio porte, capaz de resistir a grandes secas, permanecendo sempre verde, devido as suas raízes pivotantes, que permitem a obtenção de água do subsolo (Dantas et al., 2014).

É uma árvore de grande importância econômica e ecológica para a população nordestina, e possui uma carga histórica considerável, sendo utilizada em shampoos devido à presença de saponinas em várias partes da planta, na produção local de lenha e carvão, arborização de ruas e jardins, além de possuir frutos comestíveis, nos quais são explorados de forma extrativista (Lorenzi, 2009). É empregado na fabricação de sabão, cosméticos e creme dental, e na indústria madeireira (Lorenzi \& Matos, 2008), além de servir na alimentação de animais nos períodos de seca. Também é bastante explorado pela medicina popular como expectorante, no tratamento de bronquites e ulceras gástricas, além de febres, infecções bacterianas, dores, gengivite, entre outras enfermidades (Lorenzi, 2009).

A importância do estudo dessas plantas medicinais, como o Juazeiro, vem sendo apontadas pela Organização Mundial da Saúde desde 1970, como uma fonte alternativa para descoberta de novas drogas (Pan et al., 2013). Grande parte da população mundial tem acesso a plantas e ervas medicinais, até nas regiões mais pobres dos centros urbanos, são encontradas em feiras, mercados populares e nos quintais residenciais (Badke et al., 2012). Os efeitos e informações acerca de tais plantas têm sido obtidos e documentados, e demonstram o uso de várias espécies medicinais para o tratamento de várias doenças (Brito et al., 2015).

A secagem é uma técnica que permite o estudo de plantas medicinais, assim como também é capaz de aumentar a durabilidade de plantas e alimentos tendo em vista a perecibilidade dos mesmos. É uma técnica que consiste na remoção da água livre presente nos alimentos e plantas, aumentando assim sua vida útil, além de apresentar vantagens como facilidade no transporte, comercialização e redução de perdas (Celestino, 2010).

A secagem em camada de espuma (foam-mat drying) é uma técnica que transforma alimentos ou materiais líquidos em espumas estáveis, por meio de uma forte agitação e incorporação de agentes espumantes, para em seguida serem desidratados (Karim \& Cheewai, 1999). Apresenta-se como uma alternativa para reduzir a alta perecibilidade dos materiais biológicos, permitindo a manutenção da qualidade e o aproveitamento industrial do material desidratado (Nunes et al, 2018). Alguns materiais naturalmente contêm proteínas e/ou saponinas que produzem espumas quando batidos. Quando o material não produz a espuma necessariamente estável para a secagem, agentes espumantes são adicionados (Sankat \& Castaigne, 2004). 
Por possuir uma quantidade considerável de saponinas, o juazeiro se torna um material interessante para a secagem em camada de espuma, uma vez que forma espumas estáveis sem que seja necessária a adição de agentes espumantes, o que torna também interessante, o estudo do seu uso como agente espumante.

Diante do exposto, pretende-se obter o pó da entrecasca do Juazeiro (Ziziphus joazeiro Mart.) pelo método de camada de espuma, assim como fazer a análise físico-química do pó resultante, para posterior aplicação como agente espumante.

\section{Metodologia}

\subsection{Matéria prima}

A matéria-prima utilizada foram galhos de juazeiros coletados de um cultivo de árvores, localizadas no município de Lucena (Latitude: $06^{\circ} 54^{\prime} 08^{\prime \prime}$ S; Longitude: $34^{\circ} 52^{\prime} 08^{\prime \prime}$ W) situado no estado da Paraíba.

\subsection{Extração e preparação da matéria prima}

Os galhos dos juazeiros foram extraídos das árvores, ainda verdes. Em seguida, encaminhados para o preparo da matéria prima. A primeira etapa da preparação da matéria-prima (Figura 1) consistiu na limpeza dos ganhos, com o intuito de remover interferentes. Em seguida, a segunda etapa, foi a raspagem dos ganhos, a fim de remover a casca dos mesmos (parte marrom), com o auxílio de facas, expondo assim a entrecasca. Na terceira etapa da preparação, foi feita a extração da entrecasca dos galhos, obtendo assim raspas da entrecasca. Após a retirada de toda a entrecasca dos ganhos, as mesmas foram congeladas até que fossem utilizadas, a fim de aumentar a vida útil das raspas.

Figura 1 - Preparação da matéria prima: (a) limpeza, (b) remoção da casca e (c) raspagem.
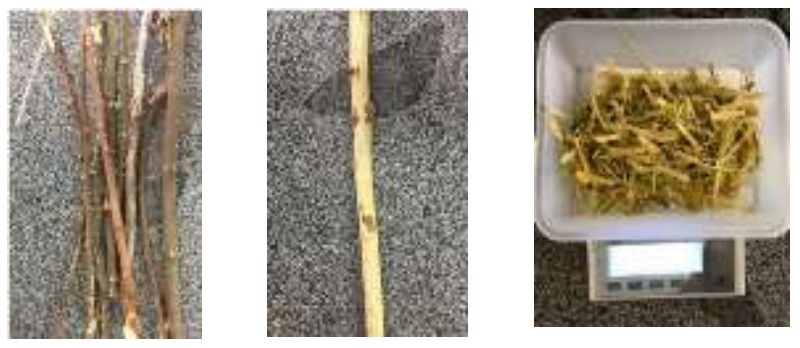

Fonte: Autores.

\subsection{Produção da espuma}

Após a extração das raspas da entrecasca dos galhos do juazeiro, três quantidades diferentes de entrecascas (15, 30 e 45 gramas) foram batidas em $150 \mathrm{~mL}$ de água, de acordo com estudos preliminares.

As entrecascas foram batidas com água em um liquidificador de alta rotação industrial da marca Metvisa, com capacidade de $2 \mathrm{~L}$ e velocidade constante de 1800 rpm, por 1 minuto. Após esse tempo o suco obtido já apresentava uma formação de espuma, dependendo da quantidade de raspa utilizada.

Após a obtenção do suco, o mesmo foi submetido à agitação mecânica vigorosa, em uma batedeira planetária da marca Arno, na velocidade 6 de agitação. De acordo com o planejamento experimental, as espumas produzidas foram obtidas com as seguintes variações: $15 \mathrm{~g} / 150 \mathrm{~mL}$ ( $10 \%$ de juazeiro - 0,1 g/mL), $30 \mathrm{~g} / 150 \mathrm{~mL}$ ( $20 \%$ de juazeiro - 0,2 g/mL) e $45 \mathrm{~g} / 150 \mathrm{~mL}$ (30\% de juazeiro - 0,3 g/mL), e variando-se o tempo de agitação em 3, 4 e 5 minutos. 


\subsection{Planejamento experimental fatorial completo}

A secagem em camada de espuma da entrecasca do juazeiro foi realizada de acordo com um planejamento experimental fatorial completo $2^{3}+3$ pontos centrais, totalizando 11 experimentos. Foram observadas a influência das variáveis independentes: tempo de agitação (tag); concentração de juazeiro (C) e temperatura de secagem (T) na variável dependente: tempo final de secagem. Nas Tabela 1 e 2 são mostrados os valores codificados e reais das variáveis independentes e a matriz do planejamento experimental fatorial completo, respectivamente.

Tabela 1 - Valores codificados e reais das variáveis independentes.

\begin{tabular}{c|c|c|c}
\hline \multirow{2}{*}{ Variáveis } & \multicolumn{3}{c}{ Níveis } \\
\cline { 2 - 4 } & -1 & 0 & +1 \\
\hline Tempo de agitação $(\mathrm{min})$ & 3 & 4 & 5 \\
Concentração de entrecasca de Juazeiro $(\mathrm{g} / \mathrm{mL})$ & 0,1 & 0,2 & 0,3 \\
Temperatura de secagem $\left({ }^{\circ} \mathrm{C}\right)$ & 50 & 60 & 70 \\
\hline
\end{tabular}

Fonte: Autores.

Tabela 2 - Matriz do planejamento fatorial completo $2^{3}+3$ pontos centrais.

\begin{tabular}{c|c|c|c}
\hline \multirow{2}{*}{ Experimentos } & \multicolumn{3}{|c}{ Variáveis independentes } \\
\cline { 2 - 4 } & $\mathrm{t}_{\mathrm{ag}}(\mathrm{min})$ & $\mathrm{C}_{\text {juá }}(\mathrm{g} / \mathrm{mL})$ & $\mathrm{T}\left({ }^{\circ} \mathrm{C}\right)$ \\
\hline 1 & $3(-1)$ & $0,1(-1)$ & $50(-1)$ \\
2 & $5(1)$ & $0,1(-1)$ & $50(-1)$ \\
3 & $3(-1)$ & $0,3(1)$ & $50(-1)$ \\
4 & $5(1)$ & $0,3(1)$ & $50(-1)$ \\
5 & $3(-1)$ & $0,1(-1)$ & $70(1)$ \\
6 & $5(1)$ & $0,1(-1)$ & $70(1)$ \\
7 & $3(-1)$ & $0,3(1)$ & $70(1)$ \\
8 & $5(1)$ & $0,3(1)$ & $70(1)$ \\
9 & $4(0)$ & $0,4(0)$ & $60(0)$ \\
10 & $4(0)$ & $0,4(0)$ & $60(0)$ \\
11 & $4(0)$ & $0,4(0)$ & $60(0)$ \\
\hline
\end{tabular}

tag - tempo de agitação; $\mathrm{C}_{\mathrm{juá}}$ - concentração de entrecasca de juazeiro; $\mathrm{T}$ - temperatura de secagem Fonte: Autores.

As variáveis independentes, assim como seus níveis, foram determinadas através de testes preliminares realizados. A fim de considerar a aleatoriedade do planejamento, os experimentos não foram feitos na mesma ordem apresentada na Tabela 2. 
O programa STATISTICA® 10 foi utilizado para o cálculo dos principais efeitos e as interações das variáveis independentes (concentração de entrecasca de juazeiro, tempo de agitação e temperatura de secagem) sobre a variável dependente (tempo final de secagem), assim como a influência das variáveis independentes sobre as características físicas da espuma e do pó obtido.

\subsection{Caracterização física da espuma da entrecasca de juazeiro}

\subsubsection{Densidade}

A densidade da espuma foi determinada através da medição da massa e do volume ocupado pela mesma em temperatura ambiente. As espumas foram colocadas em uma proveta de $100 \mathrm{~mL}$ até o preenchimento total do volume, e colocadas em uma balança semi-analítica da marca Shimadzu modelo BL3200H, sendo o cálculo da densidade feito através da Equação 1.

$$
\rho=\frac{m}{v}
$$

Sendo: $\mathrm{m}=$ massa $(\mathrm{g}) ; \mathrm{v}=$ volume $(\mathrm{mL})$

\subsubsection{Porcentagem de expansão}

Pelo cálculo das densidades do suco e da espuma foi possível determinar o percentual de expansão da espuma, através da Equação 2:

$$
\% \operatorname{expansão}=\frac{\frac{1}{\rho_{\text {espuma }}}-\frac{1}{\rho_{\text {Suco }}}}{\frac{1}{\rho_{\text {suco }}}} \times 100 \%
$$

Onde,

$\rho_{\text {suco }}=$ densidade do suco $(\mathrm{g} / \mathrm{mL})$;

$\rho_{\text {espuma }}=$ densidade da espuma $(\mathrm{g} / \mathrm{mL})$;

\subsubsection{Estabilidade}

A estabilidade da espuma foi avaliada através da metodologia de Sauter \& Montoure (1972) adaptado por Karim \& Wai (1999), onde foi medido o volume drenado de 10 gramas de espuma, em intervalos de 10 minutos, mantida nas temperaturas de secagem dos experimentos, $50^{\circ} \mathrm{C}, 60^{\circ} \mathrm{C}$ e $70^{\circ} \mathrm{C}$.

\subsubsection{Incorporação de ar (over run)}

A determinação $d a$ incorporação de ar foi feita avaliando o volume do suco do juazeiro em um béquer antes e depois da batedura. O suco preparado para cada concentração, foi submetido a forte agitação nos tempos pré-determinados no planejamento fatorial, e a expansão observada. O cálculo da incorporação de ar foi feito pela Equação 3.

$$
\text { (\%)over run }=\frac{V_{f}-V_{i}}{V_{i}} \times 100 \%
$$

Onde:

$\mathrm{V}_{\mathrm{i}}=$ Volume inicial do líquido;

$V_{f}=$ Volume da espuma formada após agitação; 


\subsection{Secagem da espuma da entrecasca de juazeiro}

A espuma obtida de acordo com o planejamento experimental fatorial completo (Tabela 2), foi depositada em bandejas de zinco galvanizado, com $1 \mathrm{~mm}$ de espessura, sem bordas laterais e com o fundo removível, previamente pesada com o auxílio de uma balança semi analítica, sendo mantida constante a espessura da espuma de $5 \mathrm{~mm}$ em todos os experimentos realizados, e colocadas em uma estufa de circulação de ar (marca Solab modelo SL102).

A bandeja contendo a espuma foi pesada em intervalos de tempos: nos primeiros 30 minutos, a bandeja foi pesada de $5 \mathrm{em} 5$ minutos, em seguida de $10 \mathrm{em} 10$ minutos, e à medida que a variação do valor obtido após a pesagem diminuía, o intervalo entre uma pesagem e outra aumentava, até não haver mais variação na massa do sistema, que é o indicativo que se atingiu o equilíbrio.

Ao final do processo, o pó obtido foi armazenado em sacos plásticos com fechamento hermético, e colocados e potes de acrílico, com fechamento hermético e sílica no seu interior para análises posteriores.

\subsection{Análises físicas e químicas dos pós obtidos}

\subsubsection{Atividade de água}

A atividade de água (aw) foi determinada pela leitura direta das amostras de pó resultante da secagem em camada de espuma no medido de água Novasina, a $30^{\circ} \mathrm{C}$.

\subsubsection{Solubilidade em água}

O método utilizado para determinar a solubilidade do pó em água foi baseado nas alterações que Cano-Chauca et al. (2005) realizaram no método de Eastman e Moore (1984). Amostras de 0,5 g de pó foram adicionados a $50 \mathrm{~mL}$ de água destilada, e submetidas a vigorosa agitação, em seguida a solução foi centrifugada e uma alíquota de 12,5 mL do sobrenadante foi colocada em uma placa de Petri, previamente tarada, e levada para estufa a $105{ }^{\circ} \mathrm{C}$ até a evaporação total da água. A solubilidade foi calculada através da Equação 4:

$$
\% \text { Solubilidade }=\frac{\left(m_{\text {residual }}-m_{\text {tara }}\right) * V_{\text {solução }}}{V_{\text {aliquota }} * m_{\text {amostra }}} * 100 \%
$$

Onde:

$\mathrm{m}_{\text {residual }}=$ massa residual após a evaporação da água (g);

$\mathrm{m}_{\text {tara }}=$ massa da placa de Petri vazia $(\mathrm{g}) ;$

$\mathrm{V}_{\text {solução }}=$ volume total da solução $(\mathrm{mL})$;

$\mathrm{V}_{\text {aliquota }}=$ volume da alíquota submetida à secagem $(\mathrm{mL}) ;$

$\mathrm{m}_{\mathrm{amostra}}=$ massa inicial da $\operatorname{amostra}(\mathrm{g})$; 


\subsubsection{Sólidos solúveis totais $\left({ }^{\circ}\right.$ brix $)$}

A medida do ${ }^{\circ}$ Brix foi realizada a temperatura ambiente $\left(25^{\circ} \mathrm{C}\right)$ com leitura direta em refratômetro Nova Instruments, colocando três gotas da solução resultante da diluição de $1 \mathrm{~g}$ do pó e $10 \mathrm{~mL}$ de água destilada submetidas a forte agitação e filtração, no equipamento e efetuando a leitura (Brasil, 2008).

\subsubsection{Potencial hdrogenionico (ph)}

$\mathrm{O}$ pH do pó foi medido através da leitura de uma solução feita com $1 \mathrm{~g}$ do pó diluído em $10 \mathrm{~mL}$ de água destilada submetidas a forte agitação e filtração, em um medidor de pH metro digital da marca Del Lab, com eletrodo de vidro, devidamente calibrado com soluções tampão de pH 7,00 e 4,00.

\subsubsection{Obtenção do extrato para identificação de saponinas}

Foi produzido um extrato dos pós a fim de realizar a identificação da presença de saponinas nas amostras de pós de juazeiro obtidos. Foram diluídos $1 \mathrm{~g}$ de pó em $50 \mathrm{~mL}$ de água destilada, a solução foi agitada por 2 minutos em um agitador de solução. Em seguida a solução foi deixada em repouso por 30 minutos e filtrada. O líquido filtrado foi colocado em béqueres de $50 \mathrm{ml}$ e colocado em uma estufa de circulação de ar (Solab modelo SL102) a $40^{\circ} \mathrm{C}$ até que ocorresse completa evaporação da água.

O extrato resultante foi encaminhado para o Laboratório Multiusuário de Caracterização e Análise (LMCAIPEFARM), onde foi colocado no equipamento.

\section{Resultados e Discussão}

\subsection{Densidade, expansão e incorporação de ar (over run)}

Os resultados para a densidade, expansão e incorporação de ar da espuma são mostrados na Tabela 3.

Através dos resultados da Tabela 3 foi observado que as densidades diminuem com a diminuição tanto da concentração de juazeiro e do tempo de agitação. Sendo o maior valor de densidade obtido para as condições do fatorial máximo ( $\mathrm{t}_{\mathrm{ag}}=5 \mathrm{~min}$; $\mathrm{C}_{\text {juá }}=30 \%$ ), e o menor valor obtido nas condições do fatorial mínimo ( $\mathrm{t}_{\mathrm{ag}}=3 \mathrm{~min} ; \mathrm{C}_{\mathrm{juá}}=10 \%$ ).

Oliveira et al (2020) ao trabalharem com a folha da gravioleira (Annona muricata Linn) utilizou o emulsificante Portogel ${ }^{\circledR}$ para a obtenção da espuma e encontrou maiores valores de densidade para menores valores de concentração de emulsificante e tempo de agitação. Na literatura, ainda não é possível encontrar dados acerca do juazeiro.

Os maiores valores de expansão foram obtidos nos experimentos 5 e 1, nas condições do fatorial mínimo do planejamento ( $\mathrm{t}_{\mathrm{ag}}=3$ minutos e $\mathrm{C}_{\mathrm{ju}}=10 \%$ ) e no ponto central. Nesse experimento a expansão foi de $1480 \%$ e $1440 \%$ respectivamente. E o menor valor obtido foi para o experimento 4 , nas condições do fatorial máximo $\left(\mathrm{t}_{\mathrm{ag}}=5\right.$ minutos e $\mathrm{C}_{\mathrm{jua}}=$ $30 \%$ ), sendo o valor da expansão de $1058 \%$. Oliveira et al (2020) encontrou resultados semelhantes para a expansão, maior expansão para menores valores de densidade.

Segundo Silva (2012) a expansão da espuma é diretamente relacionada à massa específica da mesma, ou seja, quanto maior a expansão, menor a densidade, semelhante com o que foi encontrado nesse estudo.

Para a incorporação de ar os maiores valores foram observados para os experimentos $3\left(\mathrm{t}_{\mathrm{ag}}=5\right.$ minutos e $\left.\mathrm{C}_{\text {juá }}=10 \%\right)$ e $4\left(\mathrm{t}_{\mathrm{ag}}=5\right.$ minutos e $\left.\mathrm{C}_{\text {juá }}=30 \%\right)$, tendo, ambos, $400 \%$. Os menores valores para a incorporação de ar foram obtidos nos experimentos $1\left(\mathrm{t}_{\mathrm{ag}}=3\right.$ minutos e $\left.\mathrm{C}_{\mathrm{jua}}=10 \%\right)$ e $2\left(\mathrm{t}_{\mathrm{ag}}=3\right.$ minutos e $\left.\mathrm{C}_{\mathrm{juá}}=30 \%\right)$ tendo sido, para ambos, $100 \%$ de incorporação de ar. Os experimentos com maior tempo de agitação obtiveram os maiores valores de incorporação de ar, e os menores valores para os experimentos com menor tempo de agitação. 
Tabela 3 - Valores das densidades, expansão e incorporação de ar.

\begin{tabular}{ccccc}
\hline Experimentos & Condições & Densidade $(\mathrm{g} / \mathrm{mL})$ & \%Expansão & Over run $(\%)$ \\
\hline 1 & $\mathrm{t}_{\mathrm{ag}}=3 \mathrm{~min} ; \mathrm{C}_{\text {juá }}=10 \%$ & $0,06 \pm 0,003$ & $1440 \pm 0,9$ & 100 \\
2 & $\mathrm{t}_{\mathrm{ag}}=3 \mathrm{~min} ; \mathrm{C}_{\text {juá }}=30 \%$ & $0,07 \pm 0,01$ & $1215 \pm 0,13$ & 100 \\
3 & $\mathrm{t}_{\mathrm{ag}}=5 \mathrm{~min} ; \mathrm{C}_{\text {juá }}=10 \%$ & $0,08 \pm 0,001$ & $1109 \pm 0,26$ & 400 \\
4 & $\mathrm{t}_{\mathrm{ag}}=5 \mathrm{~min} ; \mathrm{C}_{\text {juá }}=30 \%$ & $0,09 \pm 0,02$ & $1058 \pm 0,26$ & 400 \\
5 & $\mathrm{t}_{\mathrm{ag}}=4 \mathrm{~min} ; \mathrm{C}_{\text {juá }}=20 \%$ & $0,06 \pm 0,004$ & $1480 \pm 0,89$ & 100 \\
\hline
\end{tabular}

Fonte: Autores.

\subsection{Estabilidade}

As espumas que não desestabilizam ou colapsam por pelo menos uma hora, são as mais indicadas para o processo de secagem (Kudra \& Ratti, 2006). A estabilidade é determinada pelo inverso do volume drenado, ou seja, quanto menor o volume desprendido da espuma, mais estável ela é considerada, e consequentemente, melhores serão os resultados no processo de secagem (Karim \& Wai ,1999).

O comportamento da estabilidade foi analisado nas condições extremas do planejamento experimental (pontos de máximo e mínimo) e no ponto central por 90 minutos, conforme mostrado na Figura 2.

Pela Figura 2 foi observado que a espuma nas condições máximas do planejamento fatorial experimental é a que possui maior estabilidade, pois não teve volume drenado durante os 90 minutos. A espuma com menor estabilidade foi a espuma nas condições do fatorial mínimo, onde foi observado o desprendimento de $4 \mathrm{~mL}$ nos primeiros 10 minutos da análise e 5,5 mL de líquido em 90 minutos. Nas condições centrais do planejamento, ocorreu um desprendimento de 0,23 mL aos 90 minutos de análise.

As espumas que não desestabilizam ou colapsam por pelo menos uma hora, são as mais indicadas para o processo de secagem (Brygidyr, Rzepecka \& Mcconnell, 1977; Ratti \& Kudra, 2006). No estudo de Oliveira et al, (2020) foram observados o desprendimento de volume da espuma da folha da graviola de 2,5 mL, 2,6 mL e 4,4 mL para as condições mínimas, centrais e máximas do planejamento em 60 minutos, respectivamente. De modo geral, pode-se considerar o resultado de estabilidade da espuma do Ziziphus joazeiro satisfatório, visto que o maior volume drenado foi de 5,5 mL nas condições mínimas do planejamento. 
Figura 2 - Estabilidade da espuma para o Ponto Máximo (30\% de Juá; 5 minutos de agitação; 70C); Ponto Mínimo (10\% de Juá; 3 minutos de agitação; $\left.50^{\circ} \mathrm{C}\right)$; Ponto Central ( $20 \%$ de Juá; 4 minutos de agitação; $\left.60^{\circ} \mathrm{C}\right)$.

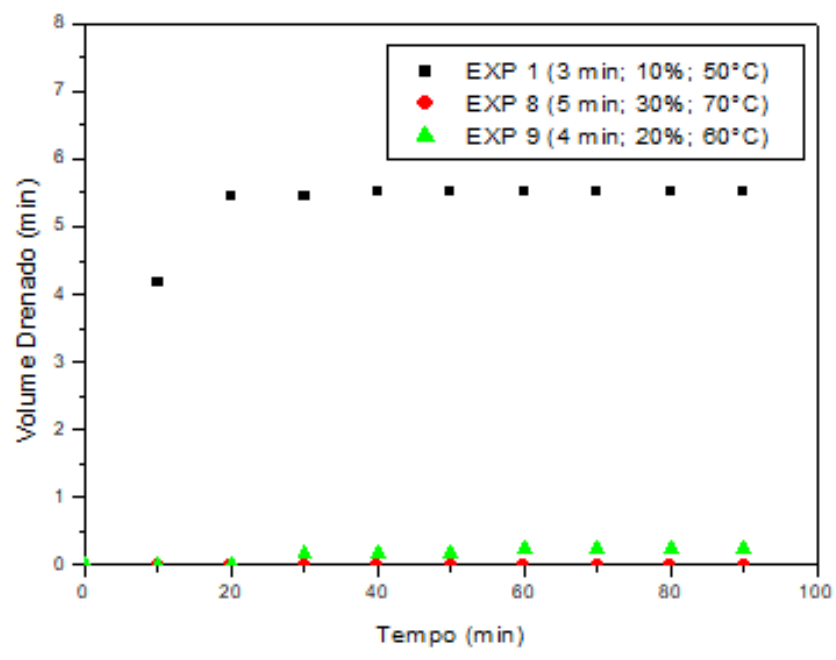

Fonte: Autores.

\section{3 secagem em camada de espuma}

Na Tabela 4 são mostrados os resultados obtidos para o tempo final de secagem da secagem das espumas da entrecasca do juazeiro.

Tabela 4 - Resultados obtidos da secagem das espumas da entrecasca do juazeiro.

\begin{tabular}{|c|c|c|c|c|}
\hline \multirow{2}{*}{ Experimentos } & \multicolumn{3}{|c|}{ Variáveis independentes } & \multirow{2}{*}{$\begin{array}{c}\text { Variável } \\
\text { dependente }\end{array}$} \\
\hline & $\mathrm{t}_{\mathrm{ag}}(\mathrm{min})$ & $\mathrm{C}_{\mathrm{juá}}(\%)$ & $\mathrm{T}\left({ }^{\circ} \mathrm{C}\right)$ & \\
\hline 1 & $3(-1)$ & $10(-1)$ & $50(-1)$ & 180 \\
\hline 2 & $5(1)$ & $10(-1)$ & $50(-1)$ & 180 \\
\hline 3 & $3(-1)$ & $30(1)$ & $50(-1)$ & 190 \\
\hline 4 & $5(1)$ & $30(1)$ & $50(-1)$ & 150 \\
\hline 5 & $3(-1)$ & $10(-1)$ & $70(1)$ & 110 \\
\hline 6 & $5(1)$ & $10(-1)$ & $70(1)$ & 120 \\
\hline 7 & $3(-1)$ & $30(1)$ & $70(1)$ & 120 \\
\hline 8 & $5(1)$ & $30(1)$ & $70(1)$ & 110 \\
\hline 9 & $4(0)$ & $20(0)$ & $60(0)$ & 130 \\
\hline 10 & $4(0)$ & $20(0)$ & $60(0)$ & 130 \\
\hline 11 & $4(0)$ & $20(0)$ & $60(0)$ & 130 \\
\hline
\end{tabular}


Pelos resultados mostrados na Tabela 4.6 foi observado que os menores tempos de secagem foram obtidos nos experimentos 5, 6,7 e 8, cuja temperatura de secagem foi de $70{ }^{\circ} \mathrm{C}$, o que reflete a influência da temperatura de secagem na secagem em camada de espuma das espumas da entrecasca do juazeiro. Notou-se ainda que, para os experimentos realizados a $50{ }^{\circ} \mathrm{C}$ teve-se os maiores tempos de secagem em relação às outras temperaturas.

\subsection{Análise estatística dos resultados da secagem}

Na Figura 3 é mostrado o Diagrama de Pareto para o tempo final de secagem da secagem das espumas da entrecasca do Ziziphus joazeiro.

Figura 3 - Diagrama de Pareto para o tempo de secagem da entrecasca do Ziziphus joazeiro.

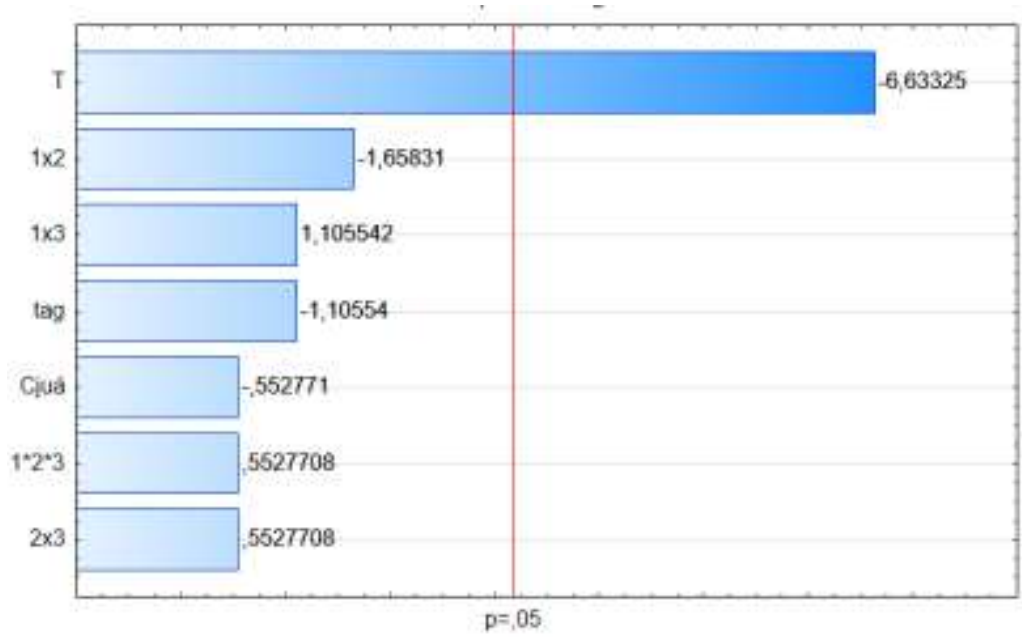

Fonte: Autores.

Pela Figura 3 foi verificado que apenas a variável independente temperatura de secagem apresentou-se significativa para o processo, exercendo uma influência de sinal negativo sobra a variável de resposta tempo de secagem, ou seja, quanto maior a temperatura, menor o tempo final de secagem.

Na Figura 4 são mostradas as superfícies de respostas que mostram a comparação entre as variáveis independentes do processo em relação a variável dependente tempo de secagem. 
Figura 4 - Superfícies de resposta para as variáveis tempo de secagem, mantendo fixo nos níveis médios: a) concentração; b) tempo de agitação; c) temperatura.

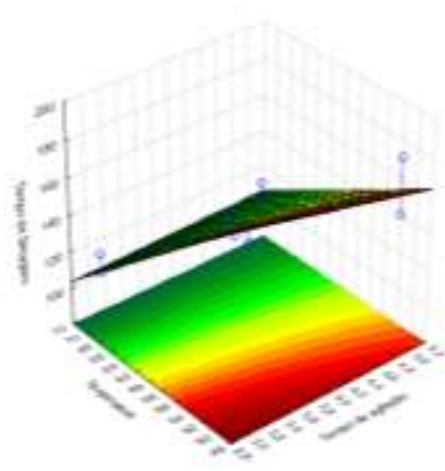

(a)

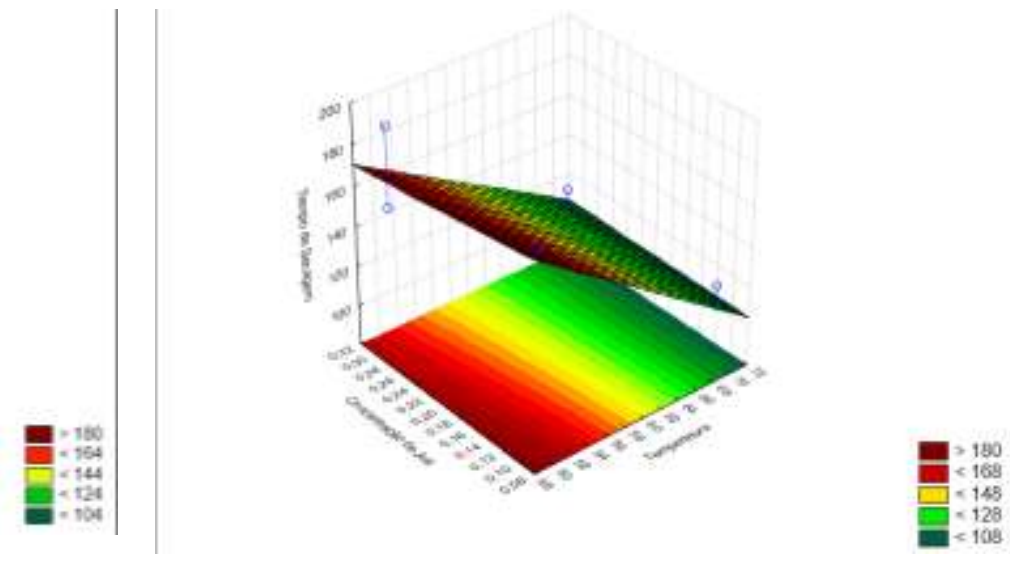

(b)

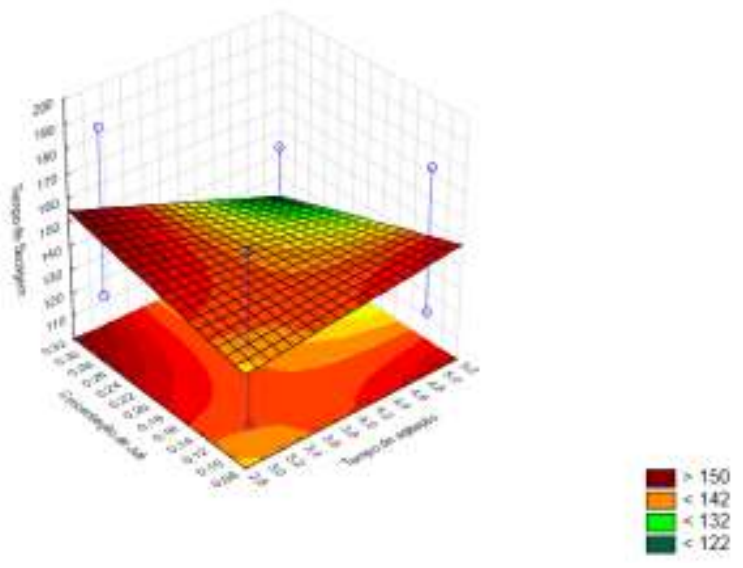

(c)

Fonte: Autores.

Pela Figura 4a foi observado que a temperatura exerce influência significativa no tempo de secagem, negativa, o que indica que quanto maior a temperatura menor será o tempo de secagem, podendo assim confirmar o resultado apresentado pelo Diagrama de Pareto (Figura 3).

É possível observar que o tempo de agitação não apresentou influência significativa nas análises, mas há uma tendencia nos maiores níveis de menor tempo de secagem, enquanto que para a concentração de juá não apresentou influência significativa sobre o tempo de secagem.

Com a análise das superfícies de respostas, é possível confirmar o resultado apresentado pelo Diagrama de Pareto (Figura 3), que mostra que a variável independente Temperatura de secagem foi a única que apresentou influência significativa no tempo de secagem, e que os menores tempos de secagem, foram obtidos nos maiores níveis de Temperatura.

\subsection{Caracterização do pó da entrecasca do juá}

Os resultados das análises feitas para caracterização do pó, atividade de água, solubilidade, ${ }^{\circ} \mathrm{Brix}$ e $\mathrm{pH}$ são apresentados na Tabela 5 . 
Tabela 5 - Resultados da atividade de água, solubilidade em água, ${ }^{\circ}$ Brix e pH do pó da entrecasca do Ziziphus joazeiro.

\begin{tabular}{c|c|c|c|c}
\hline Experimentos & aw & $\begin{array}{c}\text { Solubilidade } \\
(\%)\end{array}$ & $\mathrm{SST}\left({ }^{\circ} \mathrm{Brix}\right)$ & $\mathrm{pH}$ \\
\hline $1\left(3 \mathrm{~min}, 10 \%, 50^{\circ} \mathrm{C}\right)$ & 0,27 & $20,34 \pm 0,35$ & $18,33 \pm 0,88$ & $6,69 \pm 0,13$ \\
$2\left(5 \mathrm{~min}, 10 \%, 50^{\circ} \mathrm{C}\right)$ & 0,29 & $19,90 \pm 0,28$ & $17,33 \pm 0,44$ & $6,60 \pm 0,06$ \\
$3\left(3 \mathrm{~min}, 30 \%, 50^{\circ} \mathrm{C}\right)$ & 0,27 & $21,95 \pm 0,58$ & $20,33 \pm 1,11$ & $7,00 \pm 0,20$ \\
$4\left(5 \mathrm{~min}, 30 \%, 50^{\circ} \mathrm{C}\right)$ & 0,29 & $20,31 \pm 0,77$ & $18,33 \pm 1,11$ & $6,28 \pm 0,12$ \\
$5\left(3 \mathrm{~min}, 10 \%, 70^{\circ} \mathrm{C}\right)$ & 0,25 & $15,92 \pm 0,55$ & $18,33 \pm 0,44$ & $6,04 \pm 0,11$ \\
$6\left(5 \mathrm{~min}, 10 \%, 70^{\circ} \mathrm{C}\right)$ & 0,30 & $16,58 \pm 0,11$ & $18,83 \pm 0,22$ & $6,89 \pm 0,04$ \\
$7\left(3 \mathrm{~min}, 30 \%, 70^{\circ} \mathrm{C}\right)$ & 0,31 & $17,89 \pm 0.01$ & $17,33 \pm 1,77$ & $6,10 \pm 0,04$ \\
$8\left(5 \mathrm{~min}, 30 \%, 70^{\circ} \mathrm{C}\right)$ & 0,32 & $18,45 \pm 0,33$ & $16,33 \pm 1,11$ & $6,12 \pm 1,11$ \\
$9\left(4 \mathrm{~min}, 20 \%, 60^{\circ} \mathrm{C}\right)$ & 0,32 & $18,85 \pm 0,36$ & $20,33 \pm 0,44$ & $6,77 \pm 0,09$ \\
$10\left(4 \mathrm{~min}, 20 \%, 60^{\circ} \mathrm{C}\right)$ & 0,33 & $18,85 \pm 0,36$ & $20,33 \pm 0,44$ & $6,77 \pm 0,09$ \\
$11\left(4 \mathrm{~min}, 20 \%, 60^{\circ} \mathrm{C}\right)$ & 0,31 & $18,85 \pm 0,36$ & $20,33 \pm 0,44$ & $6,77 \pm 0,09$ \\
\hline
\end{tabular}

Fonte: Autores.

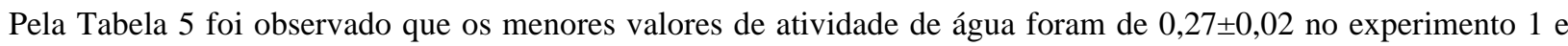
0,27 $\pm 0,01$ no experimento 3 . Foi observado que os valores foram semelhantes, não apresentando nenhuma variação considerável entre os diferentes experimentos.

Uma vez que valores de atividade de água menores que 0,3 apresentam velocidade das reações químicas e enzimáticas tendendo a zero, e como consequência o não desenvolvimento de microrganismos (Celestino, 2010), os valores obtidos nesse estudo são considerados satisfatórios, pois se encontram na faixa desejada.

A solubilidade é um parâmetro que verifica a capacidade do pó de se manter em uma mistura homogênea com água, ou seja, a estabilidade da mistura constituída pela dissolução das partículas pelo líquido (Vissoto et al., 2006).

Pelos resultados obtidos para a solubilidade em água dos pós da entrecasca do juazeiro secos em camada de espuma foi observado que os mesmos apresentaram solubilidade entre, aproximadamente, 15 e $22 \%$.

O maior valor de solubilidade obtido foi de $21,95 \pm 0,35 \%$ no experimento 3 , que possui maior concentração de entrecasca de juazeiro, menor tempo de agitação e menor temperatura de secagem. A menor solubilidade foi de $15,92 \pm 1,55 \%$ no experimento 5, que possui menor concentração de entrecasca de juazeiro, menor tempo de agitação e maior temperatura de secagem.

De acordo com a Tabela 5 foi observado pouca variação entre os valores médios de sólidos solúveis totais, sendo o maior valor encontrado no experimento 3 e no ponto central, com o valor de $20,33 \pm 1,11{ }^{\circ}$ Brix e 20,33 $\pm 0,44{ }^{\circ}$ Brix, respectivamente, e o menor valor foi no experimento 8 , com o valor de $16,33 \pm 1,11^{\circ}$ Brix. 


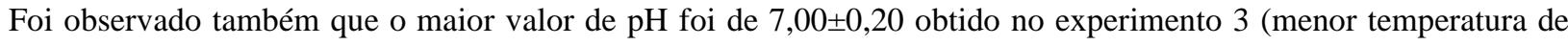
secagem e maior concentração de juazeiro). O menor valor foi de 6,04 $\pm 0,11$ obtido no experimento 5 (maior temperatura de secagem e menor concentração de juazeiro), o que indica um comportamento neutro.

\subsection{Identificação de saponinas}

Os espectros de RMN de saponinas, presentes nos pós da entrecasca de juá obtidos pela secagem em camada de espuma, são mostrados na Figura 5.

Figura 5 - Espectros de RMN de saponinas obtidos para os pós obtidos nas condições do planejamento experimental fatorial completo (oito amostras dos fatoriais e uma do ponto central totalizando nove amostras).

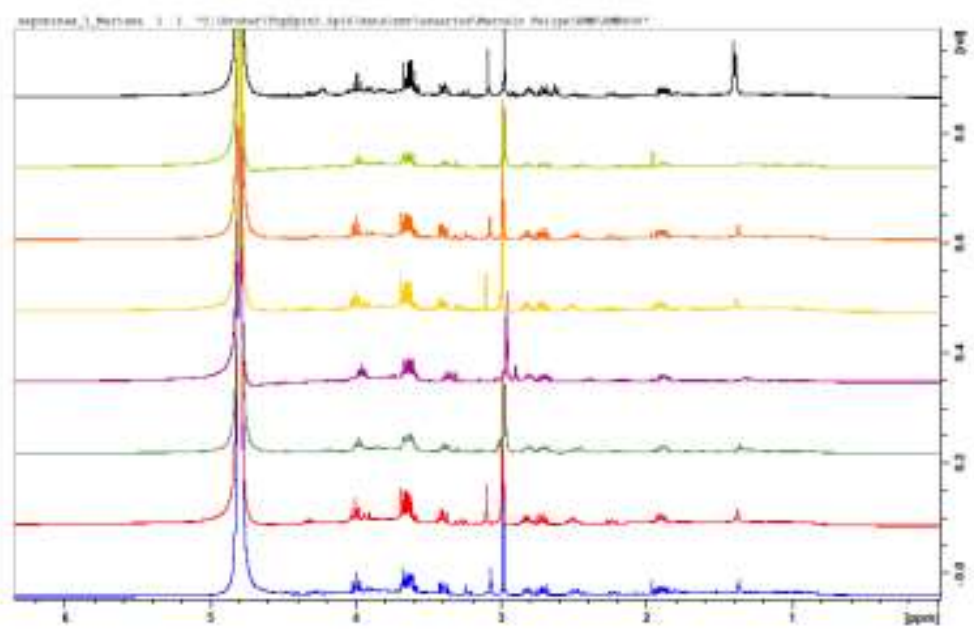

Fonte: Autores.

Khakimov et al. (2016), em seu estudo sobre triagem de saponinas em plantas, aplicou plataformas analíticas como a espectroscopia de RMN para identificar e quantificar saponinas triterpênicas em extratos de folhas de plantas Berberis vulgaris, uma planta medicinal nativa da Europa (Figura 6).

Figura 6 - Espectros de RMN obtidos para folhas da Berberis vulgares.

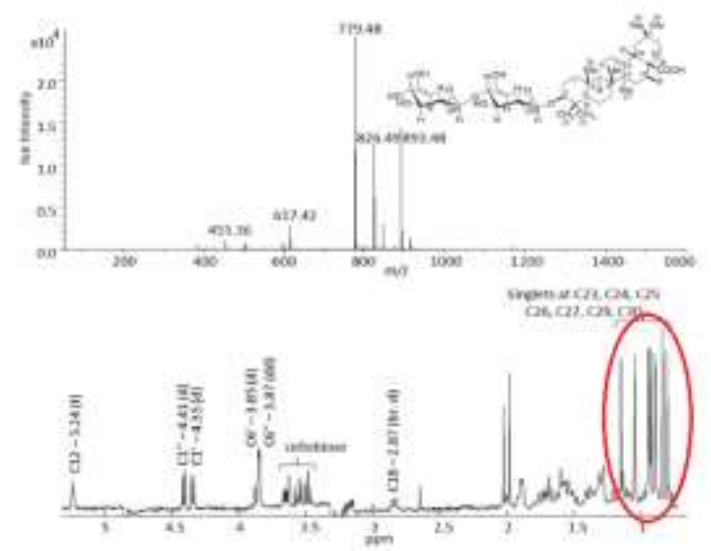

Fonte: Khakimov et al. (2016). 
O espectro mostrado na Figura 6 foi usado como comparativo para os espectros obtidos das amostras de juazeiro, uma vez que na Figura 6, na região destacada, é possível identificar os sinais bem característicos para as saponinas, sendo uma resposta a esses grupos.

Pela comparação dos espectros das Figuras 5 com a Figura 6, não é possível identificar os sinais característicos das saponinas na mesma região dos espectros do juazeiro. O que pode ser explicado pelo fato de que, saponinas, são sensíveis a altas temperaturas. Acima de $40^{\circ} \mathrm{C}$ o risco de degradação é alto. Como na secagem do juazeiro em camada de espuma, as temperaturas utilizadas foram todas acima de $40{ }^{\circ} \mathrm{C}$, isso pode ter causado uma degradação das saponinas presentes nas amostras, o que explicaria a ausência dos sinais nos espectros.

\section{Considerações Finais}

As espumas produzidas apresentaram boa estabilidade, sendo mais estáveis para maiores temperaturas de secagem e maiores concentrações de juazeiro. A densidade da espuma diminuiu com a concentração de juazeiro assim como com o tempo de agitação, sendo os valores obtidos dentro do esperado. Os menores valores de densidade resultaram em uma maior expansão da espuma, como esperado. E a maior incorporação de ar, foi obtida para o experimento com maior tempo de agitação.

Quanto a secagem a única variável que apresentou influência significativa no processo foi a temperatura, causando uma diminuição do tempo de secagem para temperatura mais elevadas.

Os valores de atividade de água do pó se mantiveram dentro da faixa considerada ideal para a conservação do produto. O maior valor de solubilidade em água foi de 20,34\% para o experimento nas condições mínimas. O teor de sólidos solúveis do pó obtidos foi considerado baixo. $\mathrm{O}$ pH das soluções produzidas com o pó obtido se encontram dentro da faixa aceitável para evitar a proliferação de microrganismos.

Não foi possível identificar os sinais característicos de saponinas nos espectros de RMN nas nove amostras.

De maneira geral, a secagem em camada de espuma mostrou-se um método satisfatório e viável para a obtenção da entrecasca do juazeiro em pó, e o pó obtido apresentou características que o apontam como um bom agente espumante, por isso para trabalhos futuros a identificação e estudos das saponinas e outros componentes que provocam a formação de espuma.

\section{Referências}

Badke, M. R., Budó M. L. D., Alvim N. A. T., Zanetti G. D, Heisler E. V. (2012). Saberes e práticas populares de cuidado em saúde com o uso de plantas medicinais. Texto contexto Enferm, 21(2), 363-70. http://www.scielo.br/pdf/tce/v21n2/a14v21n2.pdf.

Brasil. (2008). Métodos físico-químicos para análise de alimentos: normas analíticas do Instituto Adolfo Lutz (IAL). http://www.ial.sp.gov.br/resources/editorinplace/ial/2016_3_19/analisedealimentosial_2008.pdf.

Brito, S. M., Coutinho, H. D., Talvani, A., Coronel, C., Barbosa, A. G., Vega, C., Figueredo, F. G., Tintino, S. R., Lima, L. F., Boligon, A. A. (2015). Analysis of bioactivities and chemical composition of Ziziphus joazeiro Mart. Using HPLC-DAD. Food Chemistry, 186(2015), 185-191. https://doi.org/10.1016/j.foodchem.2014.10.031.

Brygidyr, A. M., Rzepecka, M. A., \& Mc Connell, M. B. (1977). Characterization and Drying of Tomato Paste Foam by Hot Air and Microwave Energy. Canadian Institute of Food Science and Technology Journal, 10(4), 313-319. https://doi.org/10.1016/S0315-5463(77)73553-9

Cano-Chauca, M., Stringheta, P. C., Ramos, A. M., \& Cal-Vidal, J. (2005). Effect of the carriers on the microstructure of mango powder obtained by spray drying and its functional characterization. Innovative Food Science and Emerging Technologies, 6, 420-428. https://doi.org/10.1016/j.ifset.2005.05.003.

Celestino, S. M. C. (2010). Princípios de Secagem de Alimentos. Embrapa Cerrados - documentos (infoteca-se).

Dantas, F. C. P., Tavares, M. L. R., Targino, M. S., Costa, A. P. (2014). Ziziphus joazeiro Mart. - Rhamnaceae: características biogeoquímicas e importância no bioma Caatinga. Revista Principia, n. v. p. 51-57.

Eastman, J. E., \& Moore, C. O. (1984). Cold water soluble granular starch for gelled food composition. U.S. Patent 4465702.

Abd Karim, A. \& Wai, C. C. (1999). Foam-mat drying of starfruit (Averrhoa carambola L.) purée. Stability and air drying characteristics. Food Chemistry, 64(3), 337-343. https://doi.org/10.1016/S0308-8146(98)00119-8. 
Research, Society and Development, v. 10, n. 12, e558101220656, 2021

(CC BY 4.0) | ISSN 2525-3409 | DOI: http://dx.doi.org/10.33448/rsd-v10i12.20656

Khakimov, B. Tseng, H. T. Godejohann, M. Søren, B. Engelsen, S. B. (2016). Screening for Triterpenoid saponins in plants usinghyphenated analytical platforms. Molecules journal, 21(12), 1614. https://doi.org/10.3390/molecules21121614.

Kudra, T., Ratti C. (2006). Foam-mat drying: Energy and cost analyses. Canadian Biosystems Engieering, 2006(48), 3.27-3.32. https://www.researchgate.net/publication/238076242.

Lorenzi, H. E. (2009). Árvores brasileiras: manual de identificação e cultivo de plantas arbóreas nativas do Brasil. 5. ed., v.1. Nova Odessa; Instituto Plantarum.

Lorenzi, H., Matos, F. J. A. (2008). Plantas medicinais no Brasil: nativas e exóticas. 2 ed. Nova Odessa: Instituto Plantarum.

Nunes, G., Nascimento, B. S., Lima-Corrêa, A. R. A. B. (2018). Avaliação da secagem em camada de espuma no aproveitamento de folhas e talos de caenoura. Blucher Chemical Enginnering Proceedings. 1(5), 662-665. https://doi.org/10.5151/cobeq2018-pt.0182

Oliveira, B. F., Negreiros, J. K. da S., Bonfim, K. S. do., Cavalcante, J. de A., Pinto, M. F., Costa, N. A. (2020). Mathematical modeling of drying kinetics in foam-mat drying of soursop (Annona muricata Linn) leaf and characterization of the obtained powder. Research, Society and Development, 9(4), p. e10942811. https://doi.org/10.33448/rsd-v9i4.2811

Pan, S., Zhou, S., Gao, S., Yu, Z., Zhang, S., Tang, M., et al. (2013). New Perspectives on How to Discover Drugs from Herbal Medicines: CAM's Outstanding Contribution to Modern Therapeutics. Evidence-Based Complementary and Alternative Medicine. http://dx.doi.org/10.1155/2013/627375. Article ID 627375.

Sankat C. K., Castaigne F. (2004). Foaming and drying behaviour of ripe bananas. LWT - Food Science and Technology, 37(5), 517-525. https://doi.org/10.1016/S0023-6438(03)00132-4.

Sauter, E. A., Montoure, J. E. (1972). The relationship of lysozyme content of egg white to volume and stability of foams. Journal of Food Science, 37(6), 918920. https://doi.org/10.1111/j.1365-2621.1972.tb03703.x.

Silva, J. E. F. V. (2012). Estudo da estabilidade das espumas da polpa de goiaba (Psidium Guajava L.). Revista Craibeiras de Agroecologia, 4(1), 55p. https://www.seer.ufal.br/index.php/era/article/view/7685

Vissotto, F. Z., Montenegro, F. M., Santos, J. M., Oliveira, S. J. R. (2006). Avaliação da influência dos processos de lecitinização e de aglomeração nas propriedades físicas de achocolatados em pó. Ciência e Tecnologia de Alimentos, 26(3), 666 - 671. 